\title{
Bistatic Synthetic Aperture Inversion for Arbitrary Flight Trajectories
}

\author{
Can Evren Yarman, Birsen Yazıcı and Margaret Cheney. \\ Rensselaer Polytechnic Institute, 110 Eighth Street, Troy, NY
}

\begin{abstract}
Reconstruction algorithms for monostatic synthetic aperture radar (SAR) with poor antenna directivity traversing straight and arbitrary flight trajectories have been developed by various authors ${ }^{1-5}$, while, to our knowledge, the acquisition geometry of bistatic SAR studies for the case of poor antenna directivity are limited to isotropic antennas traversing certain flight trajectories (straight ${ }^{6,7}$ or circular $^{8,9}$ flight trajectories) over flat topography.

In this paper, we present an approximate analytic inversion method for bistatic SAR (Bi-SAR). ${ }^{10} \mathrm{In}$ particular, we present a new filtered-backprojection (FBP) type Bi-SAR inversion method for arbitrary, but known, flight trajectories over non-flat, but known, topography. These FBP type reconstruction methods have the advantage that they produce images that have the edges of the scene at the correct location, orientation and strength. We demonstrate the performance of the new method via numerical simulations.
\end{abstract}

\section{INTRODUCTION}

In synthetic aperture radar (SAR) imaging a scene of interest is illuminated by electromagnetic waves that are transmitted from an antenna mounted on a plane or satellite. The aim is to reconstruct an image of the scene from the measurement of the scattered waves.

In bistatic SAR (Bi-SAR) ${ }^{10}$ unlike the monostatic case, where transmitter and receiver antennas are co-located, transmitter and receiver antennas are located on separate platforms (Figure 1). This allows the transmitter and its heavy power supply to be flown on a platform different from that of the cheap, expendable receiver. Also, some of the electronic countermeasures that have been devised to thwart monostatic configurations are less effective against bistatic systems. ${ }^{10-12}$ Finally, bistatic measurements can provide better ability to distinguish targets from clutter. ${ }^{13}$

For SAR systems whose antennas are able to form a narrow beam, the image reconstruction algorithms are well-known. ${ }^{14-20}$ However these algorithms are not useful for imaging systems using antennas having poor directivity where the antenna footprint is large.

$\operatorname{In}^{1-4,21}$ reconstruction algorithms for monostatic SAR with poor antenna directivity traversing straight and arbitrary flight trajectories have been developed. To our knowledge, the acquisition geometry of Bi-SAR studies for the case of poor antenna directivity are limited to isotropic antennas traversing certain flight trajectories (straight ${ }^{6,7}$ or circular $^{8,9}$ flight trajectories) over flat topography. In this paper, we focus on Bi-SAR with poor antenna directivity and address the image reconstruction problem when transmitter and receiver are traversing arbitrary, but known, flight trajectories over a known, but not necessarily flat, topography.

Further author information: (Send correspondence to C.E.Y.) C.E.Y.: E-mail: yarman@ecse.rpi.edu, Telephone: +1 (518) 2766085

B.Y.: E-mail: yazici@ecse.rpi.edu, Telephone: +1 (518) 276 2905, Fax: +1 (518) 2766261

M.C.: E-mail: cheney@rpi.edu, Telephone: +1 (518) 276 2646, Fax: +1 (518) 2764824

Algorithms for Synthetic Aperture Radar Imagery XIV, edited by Edmund G. Zelnio, Frederick D. Garber, Proc. of SPIE Vol. 6568, 656807, (2007) · 0277-786X/07/\$18 · doi: 10.1117/12.719710 


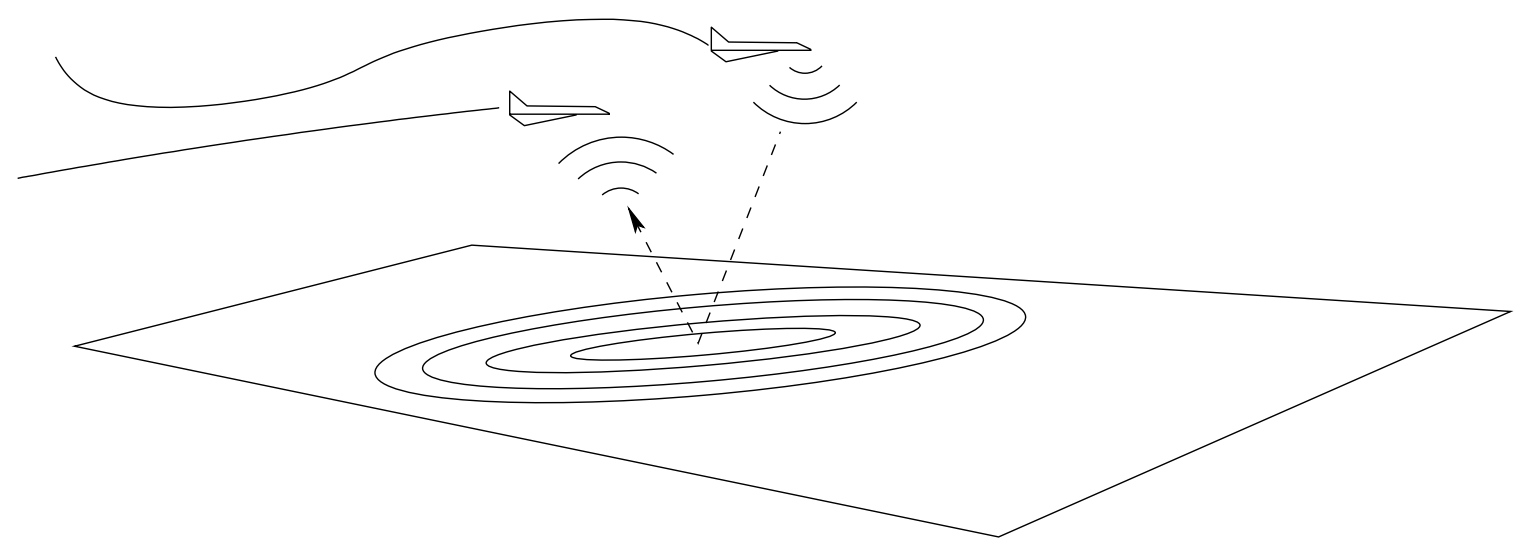

Figure 1. Acquisition geometry for Bi-SAR.

We start our discussion by introducing our forward model in Section 2. We present the FBP-type image formation method in Section 3. In Section 4, we demonstrate the performance of the image formation method in numerical simulations using circular and distorted circular flight trajectories over a flat topography. Finally, we conclude our discussion in Section 5.

\section{FORWARD MODEL}

Let $\gamma_{T}(s), \gamma_{R}(s) \in \mathbb{R}^{3}, s \in \mathbb{R}$, be the transmitter and receiver trajectories, respectively. We assume that the earth's surface is located at the position $\mathbf{x}=\left(x_{1}, x_{2}, \psi\left(x_{1}, x_{2}\right)\right)$, where $\psi: \mathbb{R}^{2} \rightarrow \mathbb{R}$, is a known smooth function, and scattering takes place in a thin region near the surface. Following, ${ }^{2,3,22}$ under the single scattering (Born) approximation, we model the received signal $d(s, t)$ as follows:

$$
d(s, t) \approx \mathcal{F}[T](s, t):=\int \mathrm{e}^{-\mathrm{i} 2 \pi \omega\left(t-R_{T R}(s, \mathbf{x}) / c_{0}\right)} A_{T R}(\boldsymbol{x}, s, \omega) T(\boldsymbol{x}) d \omega d \boldsymbol{x},
$$

where $\boldsymbol{x}=\left(x_{1}, x_{2}\right), R_{T R}(s, \mathbf{x})=\left|\gamma_{T}(s)-\mathbf{x}\right|+\left|\mathbf{x}-\gamma_{R}(s)\right|$ is the total travel time, also known as the bistatic range, ${ }^{10} T(\boldsymbol{x})$ denotes the surface reflectivity, $c_{0}$ denotes the speed of light and $\omega$ denotes the temporal frequency. $A_{T R}$ is a complex amplitude function that includes the transmitter and receiver antenna beam patterns, the transmitted waveform, geometrical spreading factors, etc. ${ }^{2,3,22}$ For example, for an isotropic transmitter transmitting a waveform $P(\omega)$ and isotropic receiver, $A_{T R}(\boldsymbol{x}, s, \omega)$ can be approximated by $A_{T R}(\boldsymbol{x}, s, \omega)=P(\omega)\left(4 \pi\left|\gamma_{T}(s)-\mathbf{x}\right|\left|\boldsymbol{\gamma}_{R}(s)-\mathbf{x}\right|\right)^{-1}$. Here $t$ denotes the time and $s$, which is referred to as the slow time, parameterizes the trajectory.

Unless otherwise stated, the bold Roman, bold italic, and Roman small letters will denote points in $\mathbb{R}^{3}, \mathbb{R}^{2}$ and $\mathbb{R}$, respectively, i.e. $\mathbf{x}=\left(\boldsymbol{x}, x_{3}\right) \in \mathbb{R}^{3}$, with $\boldsymbol{x} \in \mathbb{R}^{2}$, and $x_{3} \in \mathbb{R}$.

We assume that for some $m_{A_{T R}}, A_{T R}$ satisfies the estimate ${ }^{2,3}$

$$
\sup _{(s, \boldsymbol{x}) \in K}\left|\partial_{\omega}^{\alpha} \partial_{s}^{\beta} \partial_{x_{1}}^{\rho_{1}} \partial_{x_{2}}^{\rho_{2}} A_{T R}(\boldsymbol{x}, s, \omega)\right| \leq C_{0}\left(1+\omega^{2}\right)^{\left(m_{A_{T R}}-|\alpha|\right) / 2}
$$

where $K$ is any compact subset of $\mathbb{R} \times \mathbb{R}^{2}$, and the constant $C_{0}$ depends on $K, \alpha, \beta, \rho_{1}$, and $\rho_{2}$. This assumption is true when the antenna is broadband and the source waveform is a band-limited waveform.

In fact under the assumption (2), (1) defines $\mathcal{F}$ as a Fourier integral operator ${ }^{23-25}$ whose leading order contribution comes from those points lying in the critical points of the phase, i.e. intersection 
of the illuminated surface and the ellipsoid $\mathcal{E}(s)=\left\{\mathbf{z}: R_{T R}(s, \mathbf{z})=c_{0} t\right\}$. Since $\mathcal{F}$ is a Fourier integral operator, an approximate inverse of $\mathcal{F}$ can be computed by a suitable backprojection onto these intersections. The curves formed by the intersection of the surface and $\mathcal{E}(s)$ are also referred to as isorange contours ${ }^{10}$ for the slow-time $s$. For flat topography $\psi(\boldsymbol{x})=0$, the isorange contours are given by ellipses on the plane $x_{3}=0$ (see Figure 2), which do not necessarily share the same foci (For an explicit formulation of the ellipses $\operatorname{see}^{26}$ ).

The ideal image formation problem is to estimate $T$ from the knowledge of the data $d(s, t)$.

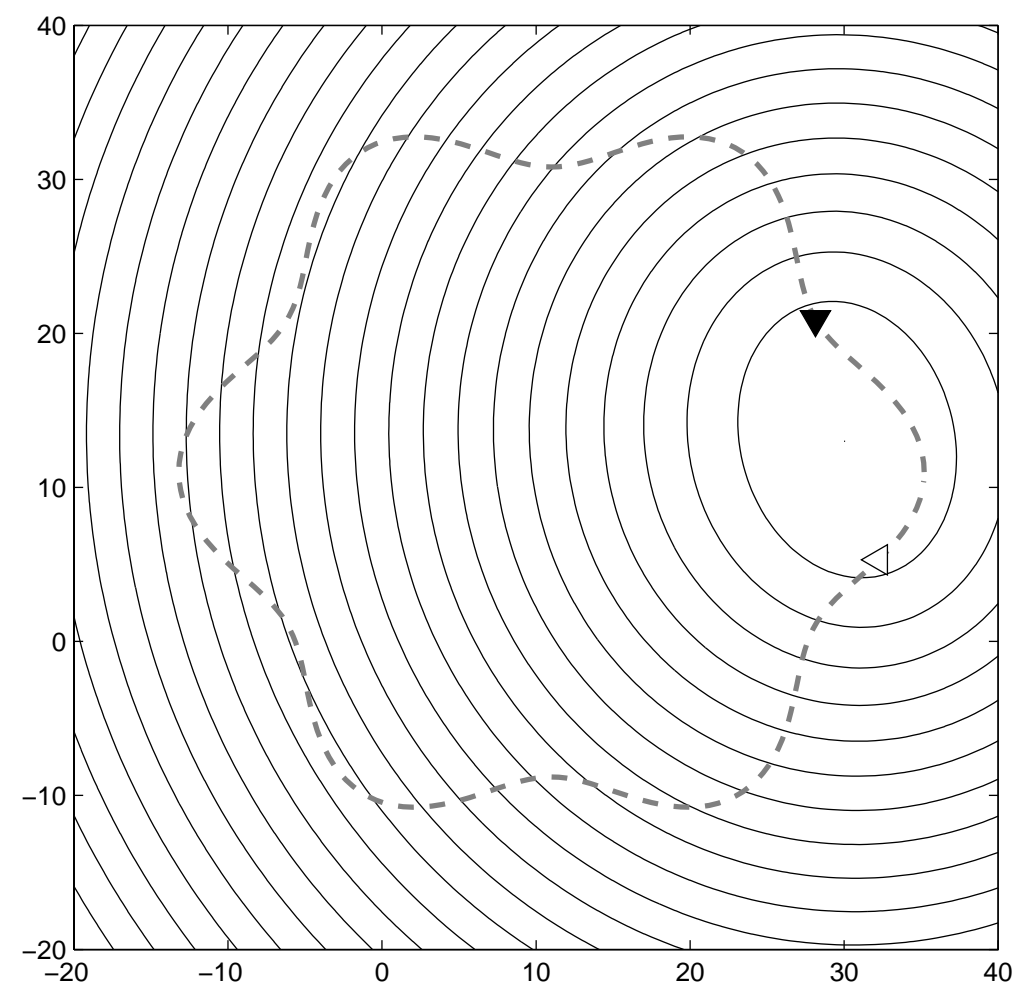

Figure 2. Isorange contour for a distorted circular flight trajectory (dashed line) $\gamma_{T}(s)=\gamma_{R}(s+\pi / 4)=\gamma_{d}(s)$ over a flat topography where black and white triangles are the transmitter and receiver platforms, respectively. (See Section 4 for an explicit formula of $\gamma_{d}(s)$.)

\section{IMAGE FORMATION}

In general, the strategy for estimating $T$ is to apply an imaging operator $\mathcal{K}$ to the data $\mathcal{F}[T]$. The image $\tilde{T}$ for the target can thus be written $\tilde{T}=\mathcal{K} \mathcal{F}[T]$. The operator $\mathcal{L}=\mathcal{K} \mathcal{F}$ contains the information about how the image $\tilde{T}$ is related to the actual target scene $T$.

Since $\mathcal{F}$ is a Fourier integral operator, an approximate inverse of $\mathcal{F}$ can be computed by another Fourier integral operator $\mathcal{K}$. Our strategy is to determine $\mathcal{K}$ so that the kernel of $\mathcal{L}$, which is also called as the point spread function, approximates the Dirac delta function. In this regard, we extend the monostatic SAR reconstruction techniques based on microlocal analysis ${ }^{2,3}$ to Bi-SAR to determine $\mathcal{K}$. We used microlocal-analysis-based methods to develop a generalized FBP-type reconstruction method where the data is first filtered and then backprojected onto isorange contours in oder to form the image. It is a direct consequence of microlocal-analysis of the backprojection operator that the visible 
edges of the scene appear in the correct location and correct orientation in the image obtained by backprojection. ${ }^{2,3}$

We use the following FBP operator $\mathcal{K}$ to form an image $\tilde{T}$ of the scene:

$$
\begin{aligned}
\tilde{T}(\boldsymbol{z}) & :=\mathcal{K}[d](\boldsymbol{z}) \\
& :=\int \mathrm{e}^{\mathrm{i} 2 \pi \omega\left(t-R_{T R}(s, \mathbf{z}) / c_{0}\right)} Q_{T R}(\boldsymbol{z}, s, \omega) d(s, t) d \omega d s d t,
\end{aligned}
$$

where $\boldsymbol{z}=\left(z_{1}, z_{2}\right), \mathbf{z}=(\boldsymbol{z}, \psi(\boldsymbol{z}))$, and

$$
Q_{T R}(\boldsymbol{z}, s, \omega)=\chi_{\Omega_{\boldsymbol{z}}}(\boldsymbol{\xi}(s, \omega)) \frac{\overline{A_{T R}(\boldsymbol{z}, s, \omega)}}{\left|A_{T R}(\boldsymbol{z}, s, \omega)\right|^{2}} \frac{1}{\eta(\boldsymbol{z}, \boldsymbol{\xi})},
$$

is the filter chosen such that the leading order term of the point spread function of $\mathcal{L}$ becomes the Dirac delta function ( $\mathrm{see}^{26,27}$ for the derivation of $Q_{T R}$ ). Here

$$
\boldsymbol{\xi}(s, \omega, \boldsymbol{z})=\frac{\omega}{c_{0}}\left[\mathrm{~J}\left(z_{1}, z_{2}\right)\right]^{T}\left[\frac{\boldsymbol{\gamma}_{T}(s)-\mathbf{z}}{\left|\gamma_{T}(s)-\mathbf{z}\right|}+\frac{\gamma_{R}(s)-\mathbf{z}}{\left|\gamma_{R}(s)-\mathbf{z}\right|}\right]
$$

where

$$
\mathrm{J}\left(z_{1}, z_{2}\right)=\left[\begin{array}{ll}
\partial \mathbf{z} / \partial z_{1} & \partial \mathbf{z} / \partial z_{2}
\end{array}\right]=\left[\begin{array}{cc}
1 & 0 \\
0 & 1 \\
\partial \psi / \partial z_{1} & \partial \psi / \partial z_{2}
\end{array}\right]
$$

and superscript ${ }^{T}$ denotes transposition,

$$
\Omega_{\boldsymbol{z}}=\left\{\boldsymbol{\xi} \mid A_{T R}(\boldsymbol{z}, s, \omega) \neq 0\right\}
$$

and $\chi_{\Omega_{z}}$ is a smooth cut-off function equal to one in the interior of $\Omega_{\boldsymbol{z}}$ and zero in the exterior of $\Omega_{\boldsymbol{z}}$, and

$$
\eta(\boldsymbol{z}, \boldsymbol{\xi})=\left|\frac{\partial(s, \omega)}{\partial \boldsymbol{\xi}}\right|
$$

is the determinant of the Jacobian that comes from the change of variables (5). We note that since $A_{T R}$ satisfies (2), by (4), for some $m_{Q_{T R}}, Q_{T R}$ satisfies the estimate. ${ }^{23-25}$

$$
\sup _{(s, \boldsymbol{x}) \in K}\left|\partial_{\omega}^{\alpha} \partial_{s}^{\beta} \partial_{x_{1}}^{\rho_{1}} \partial_{x_{2}}^{\rho_{2}} Q_{T R}(\boldsymbol{z}, s, \omega)\right| \leq C_{0}\left(1+\omega^{2}\right)^{\left(m_{Q_{T R}}-|\alpha|\right) / 2},
$$

where $K$ is any compact subset of $\mathbb{R} \times \mathbb{R}^{2}$, and the constant $C_{0}$ depends on $K, \alpha, \beta, \rho_{1}$, and $\rho_{2}$, thus making $\mathcal{K}$ a Fourier integral operator.

With the choice (4), the leading-order contribution to (3) becomes simply $\Omega_{\boldsymbol{z}}$ band-limited version of $T(\boldsymbol{z})$. An edge passing through the point $\boldsymbol{z}$ is visible if the normal to the edge is contained in $\Omega_{z} \cdot{ }^{2}$ Thus one reconstructs the visible edges of the scene with the correct strength (i.e., magnitude of the jump) and order (i.e., not smoothed). We will demonstrate these properties of the method via numerical simulations in the next section. 


\section{NUMERICAL RESULTS}

We performed numerical simulations for Bi-SAR with circular and distorted circular transmitter and receiver flight trajectories.

In our numerical simulations, we considered a square target of size $5.5 \mathrm{~km}$ and a rectangular target of size $3.3 \mathrm{~km}$ by $8.8 \mathrm{~km}$ located in a flat scene of size $[0,22] \times[0,22] \mathrm{km}^{2}$ with their centers located at $(-4.4,-2.2) \mathrm{km}$ and $(8.8,2.2) \mathrm{km}$ (see Figure 3), respectively. We discretize the scene with $128 \times 128$ pixels, where $(0,0,0) \mathrm{km}$ and $(22,22,0) \mathrm{km}$ correspond to the pixels $(1,1)$ and $(128,128)$ (see Figure 4), respectively. In these simulations, we considered the circular flight trajectory $\gamma(s)=(11+22 \cos s, 11+$ $22 \sin s, 6.5) \mathrm{km}$, and the distorted circular flight trajectory $\gamma_{d}(s)=(11+22(\cos s+.1 \cos s \cos (6 s)), 11+$ $22(\sin s+.1 \sin s \cos (6 s)), 6.5) \mathrm{km}$. For each case we took the transmitter and receiver flight trajectories to be $\gamma_{T}(s)=\gamma_{R}(s+\pi / 4)$.
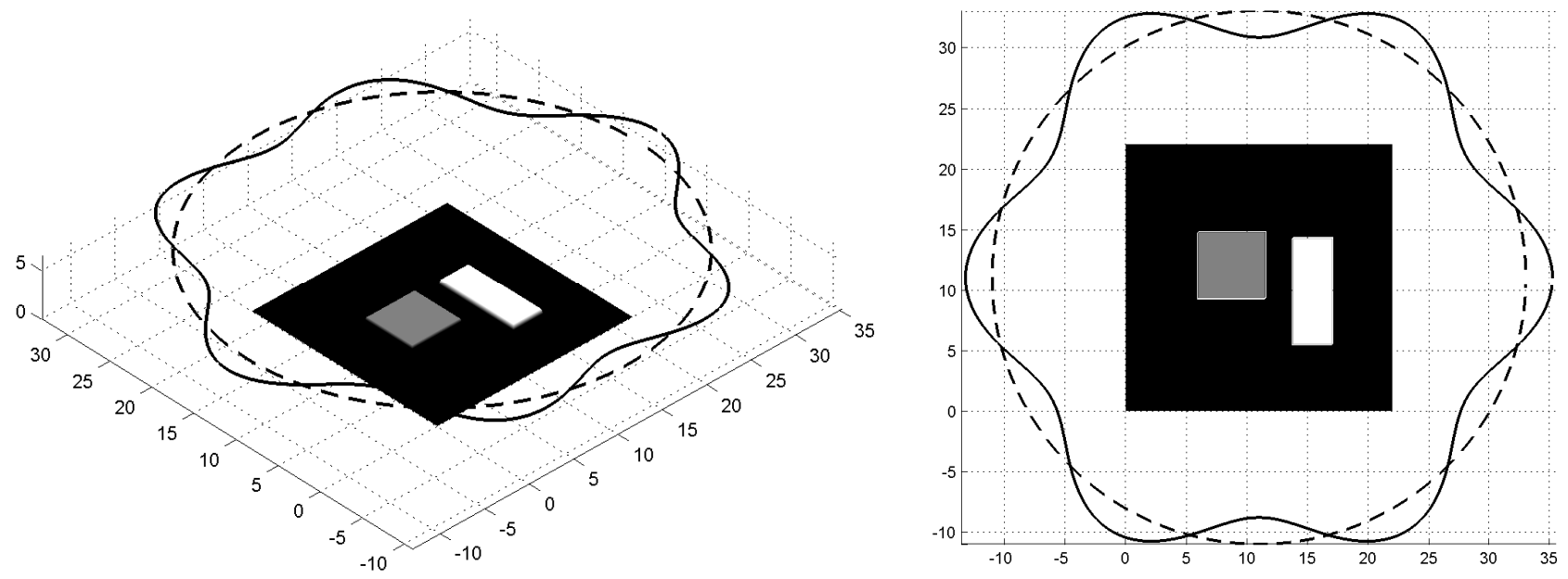

Figure 3. (Left) 3D and (right) 2D views of circular (dashed line) and distorted circular (solid line) flight trajectories.

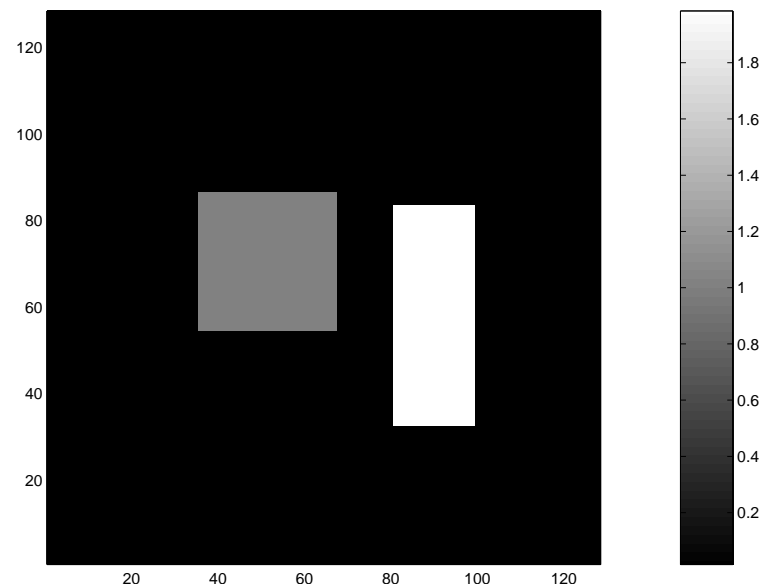

Figure 4. Scene used in numerical simulations.

For comparison purposes, we also reconstructed images using backprojection $(\mathrm{BP})^{6,9}$ only, where we set $Q_{T R}=1$. The projection data and reconstructed images using BP and FBP are presented in 
Figure 5. All the edges are visible and are reconstructed at the correct location and orientation using both the BP and FBP methods. Furthermore, the FBP method recovers the correct strength and order of the edges.
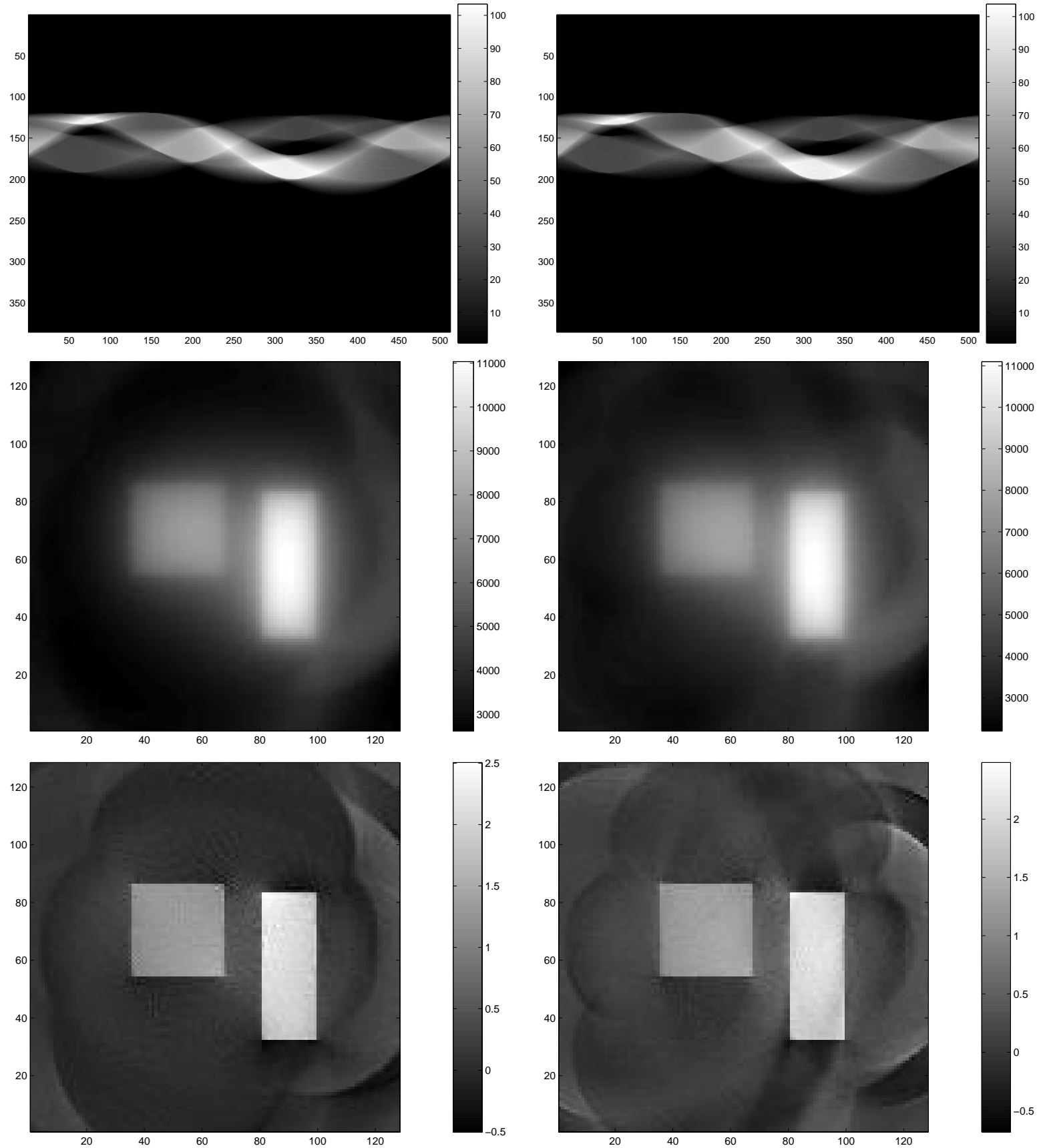

Figure 5. (Top row) Projection data for Bi-SAR with (left) circular and (right) distorted circular transmitter and receiver trajectories, with reconstructed images obtained by (middle row) backprojection and (bottom) FBP. Both methods reconstruct the visible edges of the scene at the correct location and orientation. However, the edges in the backprojected image are smoothed; this is corrected in the FBP image, as predicted by the theory. 


\section{CONCLUSION}

In this paper, we presented a new explicit filtered-backprojection-type bistatic synthetic aperture radar inversion method for arbitrary flight trajectories. The method is based on microlocal analysis and preserves the location, orientation, strength, and order of the visible edges. We demonstrated the performance of the inversion method in numerical simulations, which is in correspondence with the theoretical expectations. The method can be extended to Bi-SAR with static transmitter or receiver, ${ }^{27}$ or to multiple Bi-SARs, assuming proper data association, in a straightforward manner.

\section{ACKNOWLEDGMENTS}

We are grateful to the Air Force Office of Scientific Research for supporting this work under the agreements FA9550-04-1-0223, and FA9550-06-1-0017. Because of this support, the U.S. Government is authorized to reproduce and distribute reprints for Governmental purposes notwithstanding any copyright notation thereon. The views and conclusions contained herein are those of the authors and should not be interpreted as necessarily representing the official policies or endorsements, either expressed or implied, of the Air Force Research Laboratory or the U.S. Government.

\section{REFERENCES}

1. S. Nilsson, Application of fast backprojection techniques for some inverse problems of integral geometry. PhD thesis, Linköping Studies in Science and Technology, 1997. Dissertation No. 499.

2. C. J. Nolan and M. Cheney, "Synthetic aperture inversion," Inverse Problems 18, pp. 221-236, 2002.

3. C. Nolan and M. Cheney, "Synthetic aperture inversion for arbitrary flight paths and non-flat topography," IEEE Transactions on Image Processing 12, pp. 1035-1043, 2003.

4. L. Ulander, H. Hellsten, and G. Stenström, "Synthetic-aperture radar processing using fast factorized backprojection," IEEE Transactions on Aerospace and electronic systems 39, pp. 760-776, 2003.

5. B. Yazici, M. Cheney, and C. Yarman, "Synthetic-aperture inversion in the presence of noise and clutter," Inverse Problems 22, pp. 1705-1729, 2006.

6. M. Soumekh, "Wide-bandwidth continuous-wave monostatic/bistatic synthetic aperture radar imaging," in Proceedings of International Conference on Image Processing, 3, pp. 361-365, Oct. 1998.

7. M. Soumekh, "Bistatic synthetic aperutre radar inversion with application in dynamic object imaging," IEEE Transactions on Signal Processing 39, pp. 2044-2055, Sept. 1991.

8. J. Bauck and W. Jenkins, "Convolution-backprojection image reconstruction for bistatic synthetic aperture radar," in Proceedings of IEEE ISCAS, pp. 1512-1515, 1989.

9. S. Lockwood, A. Brown, and H. Lee, "Backward propagation image reconstruction techniques for bistaticsynthetic-aperture radar imaging systems with circular-apertureconfigurations," in Proceedings of the Thirty-Fifth Asilomar Conference on Signals, Systems and Computers, 1, pp. 110-115, Nov. 2001.

10. N. J. Willis, Bistatic Radar, Artech House, Norwood, MA, 1991.

11. W. Goj, Synthetic Aperture Radar and Electronic Warfare, Artech House, Boston, 1989.

12. A. Horne and G. Yates, "Bistatic synthetic aperture radar," in IEEE RADAR 2002, pp. 6 - 10, Oct. 2002.

13. L. Ulander and T. Martin, "Bistatic ultrawideband sar for imaging of groud targets under foliage," in Proceedings of 2005 IEEE Radar Conference, pp. 419 - 423, May 2005.

14. D. Munson, J. O'Brien, and W. Jenkins, "A tomographic formula of spotlight-mode synthetic aperture radar," in Proceedings of the IEEE, 72, pp. 917-925, Aug. 1983.

15. O. Arikan and D. M. Jr., "A tomographic formulation of bistatic synthetic aperture radar," in Proceedings of ComCon 88, p. 418, Oct. 1988.

16. J. Curlander and R. McDonough, Synthetic Aperture Radar, Wiley, New York, 1991.

17. G. Franceschetti and R. Lanari, Synthetic Aperture Radar Processing, CRC Press, New York, 1999. 
18. M. Soumekh, Synthetic Aperture Radar Signal Processing with MATLAB Algorithms, Wiley, New York, 1999.

19. L. Cutrona, Synthetic Aperture Radar. McGraw-Hill, New York, 1990.

20. C. Elachi, Spaceborne Radar Remote Sensing: Applications and Techniques, IEEE Press, New York, 1987.

21. B. Yazici and M. Cheney, "Synthetic aperture inversion for arbitrary flight paths in the presence of noise and clutter," in Proceedings of IEEE International Radar Conference, pp. 806-810, May 2005.

22. M. Cheney, "Synthetic-aperture assessment of a dispersive surface," International Journal of Imaging Systems and Technology .

23. J. J. Duistermaat, Fourier Integral Operators, Birkhauser, Boston, 1996.

24. F. Treves, Introduction to Pseudodifferential and Fourier Integral Operators, volumes I and II, Plenum Press, NY, 1980.

25. A. Grigis and J. Sjöstrand, Microlocal Analysis for Differential Operators: An Introduction, London Mathematical Society Lecture Note Series, Vol. 196, Cambridge University Press, Cambridge, 1994.

26. C. Yarman, B. Yazıcı, and M. Cheney, "Bistatic synthetic aperture radar imaging for arbitrary flight trajectories," submitted to IEEE Transactions on Image Processing , 2007.

27. C. Yarman, B. Yazıcı, and M. Cheney, "Bistatic synthetic aperture radar imaging for arbitrary flight trajectories and non-flat topography," in Proceedings of IEEE International Radar Conference, April 2007. 